\title{
LHCb RESULTS ON MULTI-PARTON INTERACTIONS, SOFT QCD AND DIFFRACTION*
}

\author{
MARCIN KUCHARCZYK \\ on behalf of the LHCb Collaboration \\ The Henryk Niewodniczański Institute of Nuclear Physics \\ Polish Academy of Sciences \\ Radzikowskiego 152, 31-342 Kraków, Poland \\ (Received November 12, 2015)

\begin{abstract}
Due to its unique pseudorapidity coverage $(2<\eta<5)$ and excellent performance, the $\mathrm{LHCb}$ detector allows for studying soft QCD processes at large rapidities and low transverse momenta. Selected results, such as charged particle multiplicities and densities, forward energy flow and prompt charm production are briefly summarized in these proceedings. The measurements are performed using large samples of proton-proton collision data accumulated with the $\mathrm{LHCb}$ detector at $\sqrt{s}=7 \mathrm{TeV}$. The results are compared with predictions from a number of different Monte Carlo event generators providing a unique insight into particle production in the forward region at the LHC.
\end{abstract}

DOI:10.5506/APhysPolBSupp.8.789

PACS numbers: 13.85.-t, 13.85.Qk, 14.70.-e

\section{Introduction}

The description of inelastic proton-proton $(p p)$ interaction is usually based on phenomenology of soft quantum chromodynamics (QCD), as soft processes cannot be completely determined via perturbative QCD due to divergences. The soft component of a collision is called the underlying event. The final state of an inelastic hadron-hadron collision can be described in Monte Carlo (MC) event generators by modelling partonic interactions, initial and final state radiation and hadronization processes. However, this requires a significant number of phenomenological parameters which are typically determined using soft QCD measurements. The present paper describes some of the key results obtained by the LHCb experiment which allow to constrain existing models of soft hadronic interactions.

${ }^{*}$ Presented at EDS Blois 2015: The $16^{\text {th }}$ Conference on Elastic and Diffractive Scattering, Borgo, Corsica, France, June 29-July 4, 2015. 
The LHCb detector [1] is a single-arm forward spectrometer covering the pseudorapidity range of $2<\eta<5$, with additional backwards coverage from a vertex detector. It is designed for the study of particles containing $b$ or $c$ quarks. This allows LHCb to make soft QCD measurements in a kinematic region that is hardly accessible by the general purpose detectors. The detector has a high-precision tracking system consisting of a silicon-strip vertex detector surrounding the $p p$ interaction region, a large-area silicon-strip detector located upstream of a dipole magnet, and three stations of silicon-strip detectors and straw drift tubes placed downstream of the magnet. Particle identification is performed by two Ring Imaging Cherenkov detectors which allow separation of charged particles in a momentum range of $2-100 \mathrm{GeV} / c$. The analyses presented in the proceedings are based on data from $p p$ collisions at centre-of-mass energy of $\sqrt{s}=7 \mathrm{TeV}$ recorded during the initial low pile-up running of the LHC with a minimum-bias trigger requiring at least one track segment in the vertex detector.

\section{Charged particle multiplicity}

One of the basic quantities sensitive to the modelling of hadronization processes is the charged particle multiplicity. Moreover, precise description of the underlying event is crucial for understanding backgrounds in beyond the Standard Model searches or precision measurements of the Standard Model parameters.

The LHCb has performed the charged particle multiplicity and density measurements using $p p$ interactions at a centre-of-mass energy of $\sqrt{s}=$ $7 \mathrm{TeV}$ with a low interaction rate. The analysis has required at least one prompt charged particle in the fiducial region of high track reconstruction efficiency, i.e. pseudorapidity range of $2.0<\eta<4.8$, momentum of $p>2 \mathrm{GeV} / c$ and transverse momentum of $p_{\mathrm{T}}>0.2 \mathrm{GeV} / c$ [2]. An event with at least one charged particle within this fiducial region is called visible. A prompt particle has been defined as a particle that either originates directly from the primary vertex or from a decay chain in which the sum of mean lifetimes is not above $10 \mathrm{ps}$. It includes daughters from weak $b / c$ decays, but not from strange decays. Only tracks originating from the luminous region of the collision and passing through the full tracking system and with a closest approach to the beam-line of less than $2 \mathrm{~mm}$ have been analysed. The tracks have been corrected for detector and reconstruction effects in order to compare the observed data to MC predictions. Additionally, the sample has been corrected for the probability that a visible event has no reconstructed tracks. The final correction accounts for reconstruction efficiencies and the unfolding for pile-up effects to produce a measure- 
ment for single $p p$ interactions. The results are compared to predictions from PYTHIA and Herwig++ which use string and cluster hadronization models respectively.

The charged particle density as a function of $\eta$ compared to MC predictions is shown in Fig. 1 (left) [2]. The data are compared to predictions by the LHCb tune of PYTHIA6, PYTHIA8.145, PYTHIA8.180 (using tune $4 \mathrm{C}$ [3]) and two Herwig++ versions with different tunes: UE-EE-4MRST with 2.6.3 and UE-EE-5-MRST with 2.7.0 [4]. All generators except the first two PYTHIA versions are tuned to LHC measurements at central rapidity. Both the non-LHC tunes significantly underestimate the charge density, while the remaining tunes describe the data well. The charged particle multiplicity integrated over $\eta$ is shown in Fig. 1 (right). The predictions by Herwig++ and PYTHIA8.180 with tune $4 \mathrm{C}$ provide a better description of the multiplicity distributions. Nevertheless, further comparisons in bins of $\eta$ and $p_{\mathrm{T}}$ [2] show that none of the considered models is able to fully describe the data in a satisfactory manner.
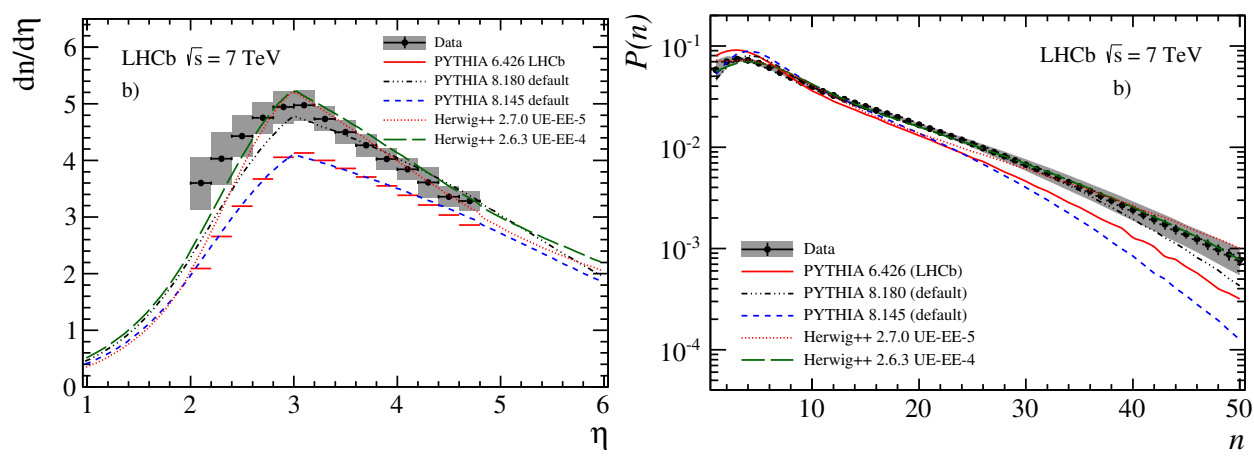

Fig. 1. Charged particle density as a function of pseudorapidity (left) and the multiplicity integrated over $\eta$ (right) [2].

\section{Forward energy flow}

The energy flow $d E_{\text {total }} / d \eta$ in high-energy hadron-hadron collisions has been measured in $\mathrm{LHCb}$ separately for different event classes allowing to probe multi-parton interactions at large $\eta$. The energy flow measured at large pseudorapidities is expected to be directly sensitive to multi-parton interaction models [5]. The measurement in $p p$ collisions at $\sqrt{s}=7 \mathrm{TeV}$ within the pseudorapidity range of $1.9<\eta<4.9$ with data recorded by the $\mathrm{LHCb}$ detector allows for constraining the underlying event models. The energy flow carried by charged particles has been extended to the measurement of the total energy flow by data-constrained Monte Carlo estimation 
of the neutral component [6]. In order to probe various aspects of multiparticle production, the measurement has been performed in four different event classes: (i) inclusive minimum bias sample with at least one track with $p>2 \mathrm{GeV} / c$ in $1.9<\eta<4.9$, (ii) hard-scattering sample having at least one track with $p_{\mathrm{T}}>3 \mathrm{GeV} / c$ in $1.9<\eta<4.9$, (iii) diffractive and, (iv) non-diffractive enriched interactions selected among the inclusive minimum-bias interactions requiring the absence or presence, respectively, of at least one backward track reconstructed by the $\mathrm{LHCb}$ vertex detector in $-3.5<\eta<-1.5$. The measured total energy flow, which is the sum of charged and neutral components, is shown for inclusive minimum bias sample in Fig. 2, superimposed with different PYTHIA versions and tunes $[7,8]$ (left), and cosmic-ray models [9] (right). The errors are dominated by systematic uncertainties, e.g. model dependence for correcting detector effects, uncertainties for the track finding and residual pile-up. The uncertainties decrease towards larger $\eta$. None of the models used in the analysis is able to describe the energy flow measurements for all event classes [6]. PYTHIA tunes in most of the cases underestimate the measurements at larger pseudorapidities but overestimate it at lower $\eta$. The majority of cosmic-ray interaction models overestimate the energy flow except for the diffractive sample. The energy flow is found to increase with the momentum transfer in an underlying $p p$ inelastic interaction.
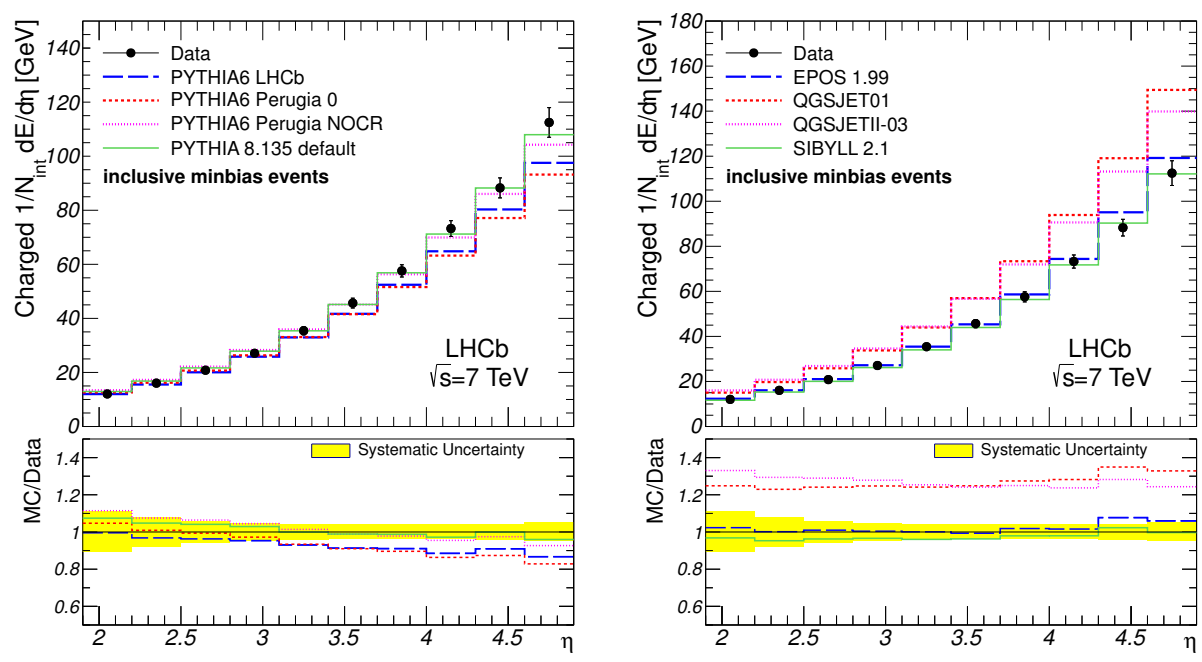

Fig. 2. Total energy flow as a function of pseudorapidity presented for inclusive minimum bias sample. The $\mathrm{LHCb}$ data is compared to different predictions from the PYTHIA (left) and cosmic ray (right) event generators [6]. 


\section{Prompt charm production}

Prompt charm production has been analysed to determine cross sections and production ratios. Production cross sections have been determined with the $\mathrm{LHCb}$ data collected in $p p$ collisions at a centre-of-mass energy of $\sqrt{s}=7 \mathrm{TeV}$ [10]. Measurements of differential production cross sections test the predictions of QCD fragmentation and hadronization models. The results of the analysis have been compared to the perturbative calculations of charmed hadron production cross sections at next-to-leading order using the Generalized Mass Variable Flavour Number Scheme (GMVFNS [11]). The results have been also cross-checked at fixed order with next-to-leading-log resummation (FONLL [12]).

The fiducial region of the measurement has been defined from $2<y<4.5$ in rapidity and up to $8 \mathrm{GeV} / c$ in transverse momentum. Prompt production of the five different charmed hadron species in bins of transverse momentum and rapidity has been analysed using the fully reconstructed decays of $D^{0} \rightarrow$ $K^{-} \pi^{+}, D^{+} \rightarrow K^{-} \pi^{+} \pi^{+}, D^{*+} \rightarrow D^{0}\left(K^{-} \pi^{+}\right) \pi^{+}, D_{s}^{+} \rightarrow \phi\left(K^{-} K^{+}\right) \pi^{+}$, $\Lambda_{c}^{+} \rightarrow p K^{-} \pi^{+}$and their charge conjugates. The prompt charmed hadrons were defined as produced at the $p p$ collision point or as fed-down from the prompt decays of excited charm resonances. Only events that have at least one reconstructed primary interaction vertex have been used, for which the selection criteria were tuned independently for each decay. Particle identification efficiencies have been determined directly from data using decays of $K_{s}^{0}, \phi$ and $\Lambda$. In order to disentangle the prompt signal yield from secondary charm contributions and combinatorial background, a multidimensional extended maximum likelihood fit has been applied to the mass and impact parameter distributions. As the $D^{*+}$ decay channel has additional sources of background related to the mismatch of the slow pion, the mass difference of the reconstructed $D^{*+}$ and $D^{0}$ has been also considered. The main sources of the systematic uncertainty may be identified as globally correlated sources (e.g. luminosity, tracking efficiency) but also correlated and uncorrelated uncertainties between bins or decay modes (e.g. particle identification efficiency, branching fractions, reconstruction and selection efficiencies).

The measured differential cross sections for the charmed mesons as a function of transverse momentum in different bins of rapidity are shown in Fig. 3. The LHCb results are in good agreement with both theoretical predictions (GMVFNS and FONLL), which successfully described the results from the Tevatron [13] and ALICE [14,15] experiments in the central rapidity region. Results of GMVFNS calculations were provided for $p_{\mathrm{T}}>3 \mathrm{GeV} / c$, as may be seen in Fig. 3. The uncertainties from scale variations in the GMVFNS were determined only for the case of $D^{0}$ production and assumed to have the same relative size for the other hadron species. In order to determine the charm hadron production ratios and total cross section in the fidu- 
cial region of $2<y<4.5$ and $0<p_{\mathrm{T}}<8 \mathrm{GeV} / c$, only the bins with uncertainty on the yield above $50 \%$ have been used. Contributions from remaining bins were extrapolated with predictions obtained from PYTHIA6.4. A combination of five individual cross sections results in a total charm cross section: $\sigma(c \bar{c})_{p_{\mathrm{T}}<8 \mathrm{GeV} / c, 2<y<4.5}=1419 \pm 12$ (stat.) \pm 116 (syst.) \pm 65 (frag.) $\mu \mathrm{b}$, where the final uncertainty is related to the fragmentation functions.
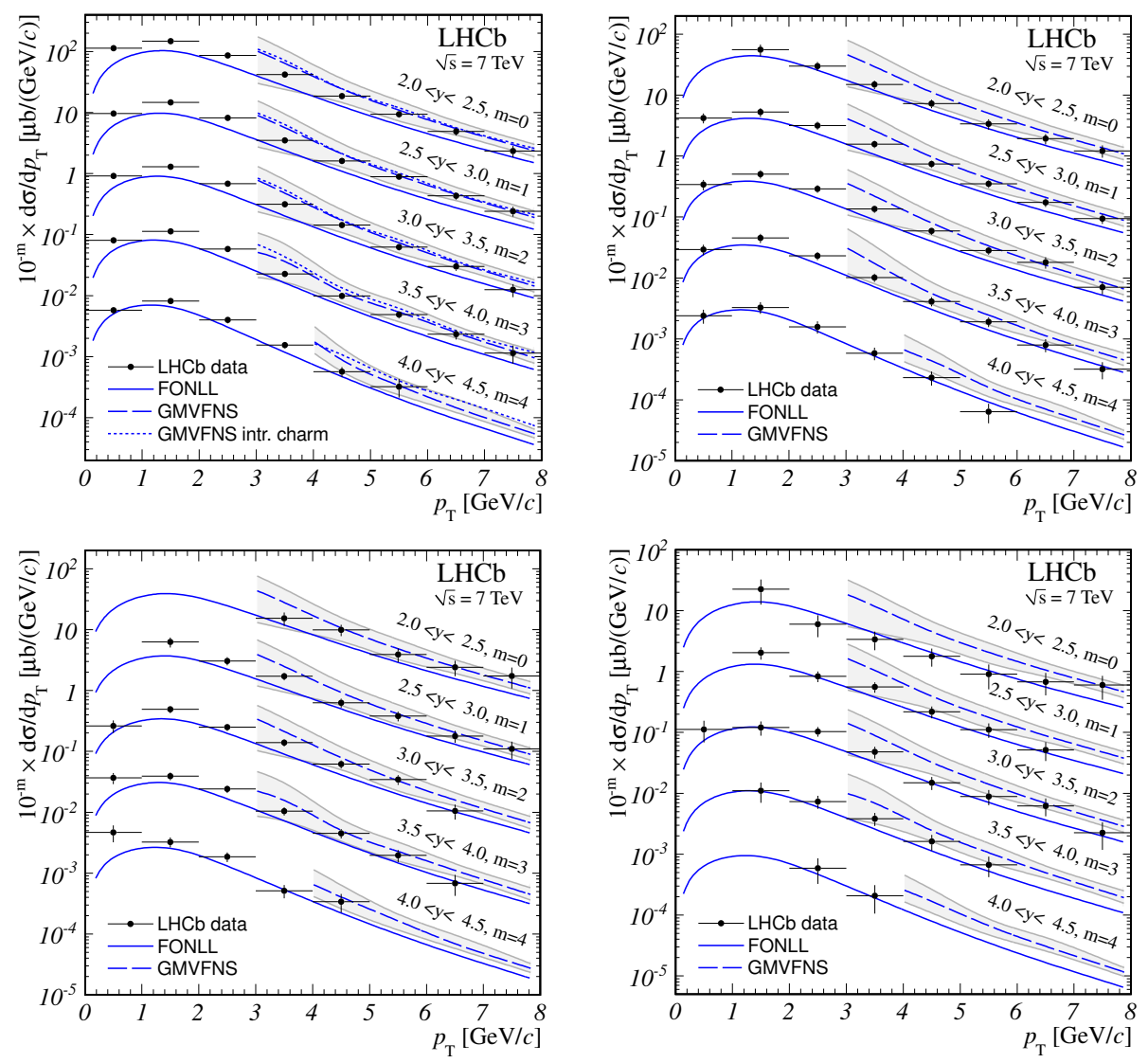

Fig. 3. Differential cross section for (from left): $D^{0}, D^{+}, D^{*+}$ and $D_{s}^{+}$, respectively [10].

\section{Summary}

$\mathrm{LHCb}$ provides complementary soft QCD measurements to the general purpose LHC detectors in the forward kinematic region. It provides important constraints for the tuning of models describing multi particle production in high energy hadronic interactions. The given results will be references for future generator optimizations, not only for the LHC but also for cosmic ray event generators. 


\section{REFERENCES}

[1] A.A. Alves Jr. et al. [LHCb Collaboration], JINST 3, S08005 (2008).

[2] R. Aaij et al. [LHCb Collaboration], Eur. Phys. J. C 73, 2421 (2013).

[3] R. Corke, T. Sjostrand, J. High Energy Phys. 1103, 032 (2011).

[4] S. Gieseke, C. Rohr, A. Siodmok, Eur. Phys. J. C 72, 2225 (2012).

[5] T. Sjostrand, M. van Zijl, Phys. Rev. D 36, 2019 (1987).

[6] R. Aaij et al. [LHCb Collaboration], Eur. Phys. J. C 74, 2888 (2014).

[7] P. Skands, Phys. Rev. D 82, 074018 (2010).

[8] T. Sjostrand et al., Comput. Phys. Commun. 178, 852 (2008).

[9] D. d'Enterria et al., Astropart. Phys. 35, 98 (2011).

[10] R. Aaij et al. [LHCb Collaboration], Nucl. Phys. B 871, 1 (2013).

[11] B. Kniehl et al., Eur. Phys. J. C 72, 2082 (2012).

[12] M. Cacciari et al., J. High Energy Phys. 1210, 137 (2012).

[13] D. Acosta et al. [CDF Collaboration], Phys. Rev. Lett. 91, 241804 (2003).

[14] B. Abelev et al. [ALICE Collaboration], J. High Energy Phys. 1207, 191 (2012).

[15] B. Abelev et al. [ALICE Collaboration], Phys. Lett. B 718, 279 (2012). 